\title{
Discovering New Physics in the Decays of Black Holes
}

\author{
Greg Landsberg \\ Department of Physics, Brown University, Providence, RI 02912, USA
}

\begin{abstract}
If the scale of quantum gravity is near a $\mathrm{TeV}$, the $\mathrm{LHC}$ will be producing one black hole (BH) about every second, thus qualifying as a BH factory. With the Hawking temperature of a few hundred $\mathrm{GeV}$, these rapidly evaporating BHs may produce new, undiscovered particles with masses $\sim 100$ $\mathrm{GeV}$. The probability of producing a heavy particle in the decay depends on its mass only weakly, in contrast with the exponentially suppressed direct production. Furthemore, BH decays with at least one prompt charged lepton or photon correspond to the final states with low background. Using the Higgs boson as an example, we show that it may be found at the LHC on the first day of its operation, even with incomplete detectors.
\end{abstract}

PACS numbers: 04.70, 04.50, 14.80.-j

Introduction: An exciting consequence of TeV-scale quantum gravity [1] is the possibility of production of black holes (BHs) [2, 3, 4] at CERN's Large Hadron Collider (LHC) and beyond. As was shown recently [5, 6, the cross section fo $\mathrm{BH}$ production at the $\mathrm{LHC}$ is $\sim 100 \mathrm{pb}$ for a fundamental Planck scale $\left(M_{P}\right) \sim 1 \mathrm{TeV}$, which would turn the LHC into a BH factory with a production rate $\sim 1 \mathrm{~Hz}$.

Once produced, the $\mathrm{TeV}$ BHs quickly evaporate via Hawking radiation [7] into half-a-dozen of particles [5, 6. Since gravity couples "democratically" to different particle species, BHs would evaporate predominantly into particles on our brane $[$, with relative probabilities of emitting different particles depending mainly on particle quantum numbers (e.g., spin and color), and only slightly on particle mass, as long as it is below the Hawking temperature. For a typical $\mathrm{BH}$ with the mass $\sim 1 \mathrm{TeV}$, the Hawking temperature is a few hundred $\mathrm{GeV}$ [5], which opens the possibility of producing new particles with masses $\sim 100 \mathrm{GeV}$ in decays of BHs.

This has exciting consequences for searches for new physics at the LHC and beyond, as the production cross section for any new particle via this mechanism is (i) large, and (ii) depends only weakly on particle mass, in contrast with the exponentially suppressed direct production mechanism. In this Letter, we consider a search for an intermediate-mass Higgs boson, most challenging to observe at the Tevatron [8], as an exciting example of this possibility. We show that for $M_{P} \sim 1 \mathrm{TeV}$, a 130 $\mathrm{GeV}$ Higgs boson can be observed in decays of $\mathrm{BHs}$ at the LHC in as little as one hour of operation, even with incomplete detectors.

Black Hole Production and Decay: The partonlevel black hole production cross section is given by [5:

$$
\sigma\left(M_{\mathrm{BH}}\right) \approx \pi R_{S}^{2}=\frac{1}{M_{P}^{2}}\left[\frac{M_{\mathrm{BH}}}{M_{P}}\left(\frac{8 \Gamma\left(\frac{n+3}{2}\right)}{n+2}\right)\right]^{\frac{2}{n+1}},
$$

where $n$ is the number of large extra dimensions, $R_{S}$ is the Schwarzschild radius of the $\mathrm{BH}$, and $M_{\mathrm{BH}}$ is its mass. In order to obtain the production cross section in $p p$ collisions at the LHC, we use the parton luminosity approach [5, 6, 9]:

$$
\frac{d \sigma(p p \rightarrow \mathrm{BH}+X)}{d M_{\mathrm{BH}}}=\left.\frac{d L}{d M_{\mathrm{BH}}} \hat{\sigma}(a b \rightarrow \mathrm{BH})\right|_{\hat{s}=M_{\mathrm{BH}}^{2}},
$$

where the parton luminosity $d L / d M_{\mathrm{BH}}$ is defined as the sum over all the types of initial partons:

$$
\frac{d L}{d M_{\mathrm{BH}}}=\frac{2 M_{\mathrm{BH}}}{s} \sum_{a, b} \int_{M_{\mathrm{BH}}^{2} / s}^{1} \frac{d x_{a}}{x_{a}} f_{a}\left(x_{a}\right) f_{b}\left(\frac{M_{\mathrm{BH}}^{2}}{s x_{a}}\right),
$$

and $f_{i}\left(x_{i}\right)$ are the parton distribution functions (PDFs). We used the MRSD $-^{\prime}$ [10] PDFs with the $Q^{2}$ scale taken to be equal to $M_{\mathrm{BH}}$, which is within the allowed range of these PDFs for up to the kinematic limit at the LC. The dependence of the cross section on the choice of PDF is $\sim 10 \%$.

As we expect unknown quantum gravity effects to play an increasingly important role for the BH mass approaching the fundamental Planck scale, following the prescription of Ref. [5], we do not consider BH masses below the Planck scale. It is expected that the $\mathrm{BH}$ production rapidly turns on, once the relevant energy threshold $\sim M_{P}$ is crossed. (At lower energies, we expect $\mathrm{BH}$ production to be exponentially suppressed due to the string excitations or other quantum effects.)

The total production cross section for $M_{\mathrm{BH}}>M_{P}$ at the LHC, obtained from Eq. (1), ranges between $15 \mathrm{nb}$ and $1 \mathrm{pb}$ for the Planck scale between $1 \mathrm{TeV}$ and $5 \mathrm{TeV}$, and varies by $\approx 10 \%$ for $n$ between 2 and 7 .

Once produced, the $\mathrm{BHs}$ evaporate at the Hawking temperature $T_{H}$ given by [11]:

$$
T_{H}=M_{P}\left(\frac{M_{P}}{M_{\mathrm{BH}}} \frac{n+2}{8 \Gamma\left(\frac{n+3}{2}\right)}\right)^{\frac{1}{n+1}} \frac{n+1}{4 \sqrt{\pi}}
$$

As the parton collision energy increases, the resulting BH gets heavier and its decay products get colder. The average multiplicity of particles produced in the $\mathrm{BH}$ evaporation is given by [5]: $\langle N\rangle \approx \frac{M_{\mathrm{BH}}}{2 T_{H}} \sim 5$. Note, that due to 
the rapidly falling $\mathrm{PDF}$, most of the $\mathrm{BHs}$ are produced with $M_{\mathrm{BH}} \approx M_{P}$, and therefore the Hawking temperature given by Eq. (2) increases roughly proportional to the Planck mass; consequently, the average decay multiplicity stays approximately constant.

The decay of a BH is thermal: it obeys all local conservation laws, but otherwise does not discriminate between particle species. Since the typical decay involves a large number of particles, the $\mathrm{BH}$ emits all the $\approx 60 \mathrm{SM}$ particles with roughly equal probability. Since there are six charged leptons and one photon, we expect $\sim 10 \%$ of the decay products to be hard, primary charged leptons and $\sim 5 \%$ of them to be hard photons or $W / Z$ bosons, each carrying hundreds of GeV of energy. Similarly, approximately $75 \%$ of particles produced in $\mathrm{BH}$ decays are quarks and gluons (due to the color-factor enhancement), while $\sim 1 \%$ of them are Higgs bosons, provided that they are sufficiently light.

A relatively large fraction of prompt and energetic photons, electrons, and muons are expected in the highmultiplicity $\mathrm{BH}$ decays, which would make it possible to select pure samples of $\mathrm{BH}$ events, which are also easy to trigger on [5, 6]. These can be used to search for other new particles that could appear in the $\mathrm{BH}$ decays at rates similar to those for the SM species.

Higgs Boson Production in the Black-Hole Decays: As an example, we use the production of a SM-like Higgs boson with the mass of $130 \mathrm{GeV}$, still allowed in low-scale supersymmetry models, but very hard to establish at the Fermilab Tevatron [8]. We consider decay of the Higgs boson into pair of jets, dominated by the $b \bar{b}$ final state $(57 \%)$, with an additional $10 \%$ contribution from the $c \bar{c}, g g$, and hadronic $\tau \tau$ final states.

We model the production and decay of the $\mathrm{BH}$ with the TRUENOIR Monte Carlo generator [12], which implements a euristic algorithm to describe a spontaneous decay of a BH. The generator is interfaced with the PYTHIA Monte Carlo program 13 to account for the effects of initial and final state radiation, particle decay, and fragmentation. We used a $1 \%$ probability to emit the Higgs particle in the $\mathrm{BH}$ decay, which is likely to be a conservative estimate, as the emission of a spin- 0 particle is expected to be enhanced by the grey body factors [14. While the multidimensional grey body factors are yet to be calculated, the 4-dimensional analysis shows that the emission of the Higgs boson can be enhanced by as much as a factor of 2.5 15.

We reconstruct electron, muons, photons, and jets within the rapidity range of \pm 2.5 and require the transverse energy above $50 \mathrm{GeV}$ for leptons and photons, and $25 \mathrm{GeV}$ for jets. Jets are reconstructed using a partonlevel clustering algorithm and then smeared assuming energy resolution $\sigma(E) / E=0.60 / \sqrt{E / \mathrm{GeV}} \oplus 0.02$ [16], typical of an LHC detector. Note, that the high boost of the particles produced in the $\mathrm{BH}$ decay with the average energy $\sim T_{H}$ improves the dijet mass resolution. For the studies that involve $b$-tagging, we assume the tagging efficiency of $60 \%$, with a $5 \%$ probability of mistakenly tagging a non- $b$ jet; this high mistagging probability reflects an enhanced charm quark content in the BH decays.

In order to select a pure sample of $\mathrm{BH}$ events, we used the prescription of Ref. [5] by selecting only the events with four or more objects (i.e., electrons, muons, photons, or jets) in the final state, with at least one of them being not a jet. This selection reduces SM backgrounds to a negligible level, see Fig. 2 in Ref. [5], and results in a typical signal acceptance of $7 \%$, dominated by the probability to find an isolated prompt lepton or photon in the event. (A Higgs boson is found in about $5 \%$ of these tagged events.)

The dijet invariant mass spectrum in the decays of BHs with the masses above $M_{P}=1 \mathrm{TeV}$ is shown in Fig. 1 . The three panes correspond to all jet combinations (with the average of approximately four jet combinations per event), combinations with at least one $b$-tagged jet, and combinations with both jets $b$-tagged. The most prominent feature in all three plots is the presence of three peaks with the masses around 80, 130, and $175 \mathrm{GeV}$. The first peak is due to the hadronic decays of the $W$ and $Z$ bosons produced in the $\mathrm{BH}$ decay either directly or in the decays of the top and Higgs particles. (The resolution of a typical LHC detector does not allow to resolve $W$ and $Z$ in the dijet mode.) The second peak is due to the $h \rightarrow j j$ decays, and the third peak is due to the $t \rightarrow W b \rightarrow j j b$ decays, where the top quark is highly boosted. In this case, one of the jets from the $W$ decay sometimes overlaps with the prompt $b$-jet from the top quark decay, and thus the two are reconstructed as a single jet; when combined with the second jet from the $W$ decay, this gives a dijet invariant mass peak at the top quark mass. The data set shown in Fig. 1 consists of $50 \mathrm{~K} \mathrm{BH}$ events, which, given the $15 \mathrm{nb}$ production cross section, corresponds to an integrated luminosity of $3 \mathrm{pb}^{-1}$, or less than an hour of the LHC operation at the nominal luminosity.

We calculate the signal significance via a counting experiment in the optimal window of $\pm 2 \sigma$ around the signal Gaussian [17]. Given the signal $S$ and the background $B$ in this window, we define the significance as $S / \sqrt{B+(\delta B)^{2}}$, where $\delta B$ is the uncertainty on the background, as determined from the fit. The significance of the Higgs signal shown in Fig. 1 a is $6.7 \sigma$.

The effect of $b$-tagging is shown in the other two panes of Fig. 1. Only the jet pair combinations with at least one of the two jets tagged are shown in Fig. 1 b; the pairs with both jets tagged are shown in Fig. 11c. While $b$-tagging improves the signal-to-background ratio, the Higgs signal significance with a single or double $b$-tagging deteriorates slightly $(6.6 \sigma$ and $5.4 \sigma$, respectively). The possibility to discover the Higgs boson in the $b \bar{b}$ decays without $b$-tagging has an important impact on the LHC collaborations, as the vertex detector installation is likely to be 

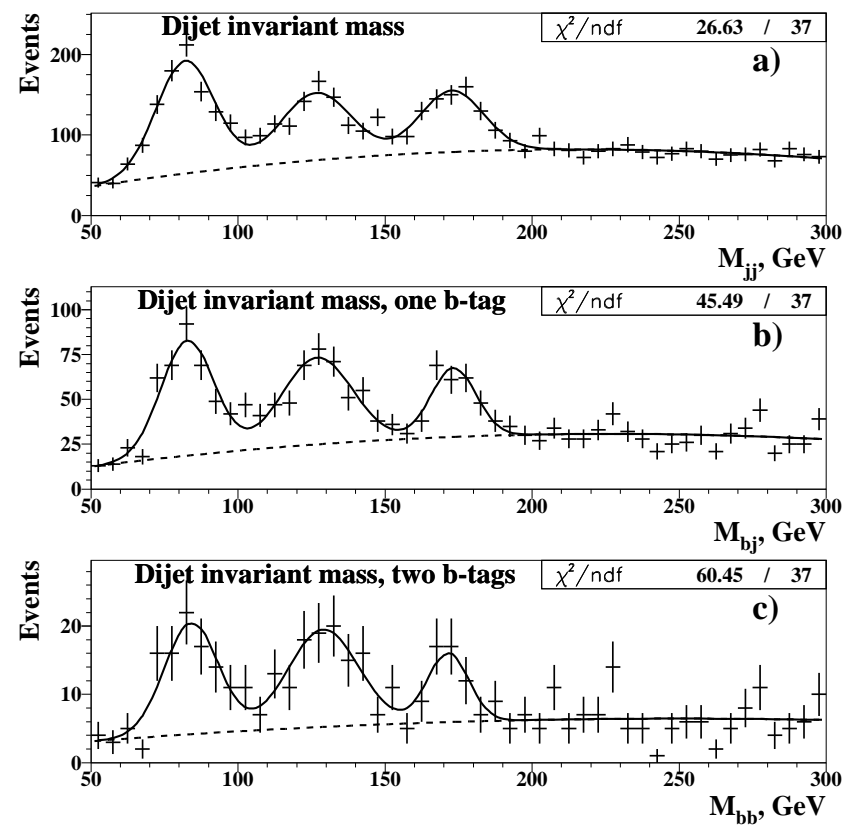

FIG. 1: Dijet invariant mass observed in the BH decays with a prompt lepton or photon tag in $\approx 3 \mathrm{pb}^{-1}$ of the LHC data, for $M_{P}=1 \mathrm{TeV}$ and $n=3$ : (a) all jet combinations; (b) jet combinations with at least one of the jets tagged as a $b$-jet; (c) jet combinations with both jets tagged as $b$-jets. The solid line is a fit to a sum of three Gaussians and a polynomial background, shown with the dashed line. The three peaks correspond to the $W / Z$ bosons, the Higgs boson, and the top quark (see text). The $\chi^{2}$ per d.o.f. is shown to demonstrate the quality of the fit. Note, that as the maximum likelihood fit was used for all cases, the $\chi^{2}$ in (c) is not an appropriate measure of the fit quality due to low statistics. Using the Poisson statistics, the probability of the fit (c) is $8 \%$.

staged. Moreover, even with the installed detectors, it takes a large amount of data and a long time to understand and align them, so that high $b$-tagging efficiency can be achieved. One would not expect this to happen before several months of stable running of the LHC. On the other hand, the discovery in the non-tagged channel can happen almost immediately after the LHC turn-on, using preliminary calibrated calorimeters only.

Another way to improve the Higgs boson identification is to select only the events with a low amount of missing energy, which guarantees that the jets are not grossly mismeasured. The effect of a $\mathbb{E}_{T}<50 \mathrm{GeV}$ cut is shown in Fig. 2, based on 50K BH events for $M_{P}=2 \mathrm{TeV}$ and $n=3$, which corresponds to $\sim 100 \mathrm{pb}^{-1}$ of data, given the $452 \mathrm{pb}$ production cross section. The top pane is the same as in Fig. 17a, while the bottom pane shows a slight improvement in the signal-to-background ratio after the $\mathbb{E}_{T}$ cut is applied.

In order to find Higgs discovery significance as a function of the integrated luminosity $(\mathcal{L})$, we use the above significance definition. Both the signal and background are scaled with the integrated luminosity. Since the background can be determined from the dijet mass distribu-
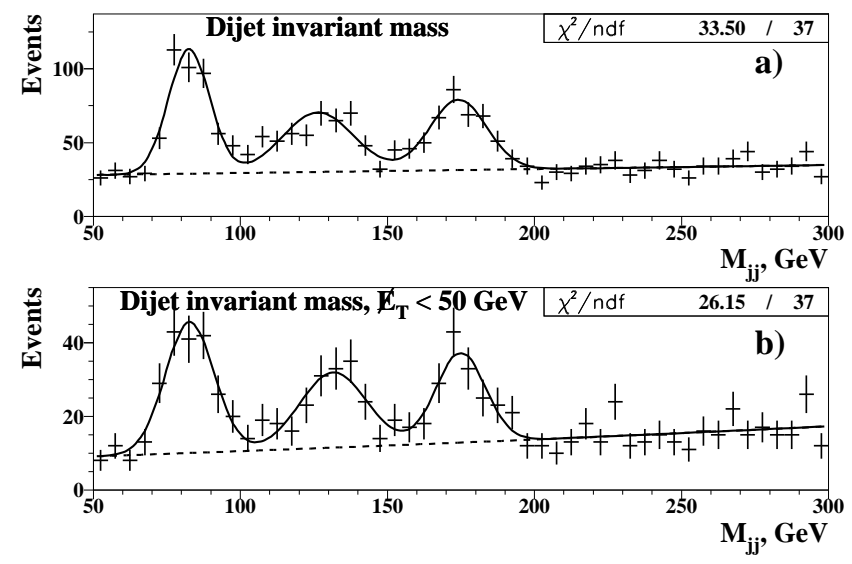

FIG. 2: Dijet invariant mass observed in the BH decays with a prompt lepton or photon tag in $\approx 100 \mathrm{pb}^{-1}$ of the LHC data, for $M_{P}=2 \mathrm{TeV}$ and $n=3$ : (a) all jet combinations; (b) same, for a subset of the events with $\mathbb{E}_{T}<50 \mathrm{GeV}$. See Fig. 1 caption for explanation.

tion away from the signal region, we assume that the error on the background is dominated by the statistics and scales as $1 / \sqrt{\mathcal{L}}$, until it becomes limited by the systematics due to the shape of the fitting function, which is taken to be $5 \%$. Constant fractional systematic uncertainty results in a plateau in signal significance, as its luminosity dependence cancels out when the systematics dominates. The results are shown in Fig. 3 for several choices of $M_{P}$, between 1 and $5 \mathrm{TeV}$.

The effect of $b$-tagging is shown in Fig. 3 with the family of curves that correspond to $M_{P}=1 \mathrm{TeV}$. While the data without $b$-tagging (the leftmost curve) gives a slightly higher significance, it reaches the plateau at lower values of the significance than the single $b$-tag (middle curve) and double $b$-tag (the rightmost curve) data. This is due to a higher signal-to-background ratio in the tagged sample. The effect of a $E_{T}<50 \mathrm{GeV}$ cut is illustrated with the two curves that correspond to $M_{P}=2 \mathrm{TeV}$. Again, while the significance is slightly higher for the full sample, the saturation is achieved at higher values for the sample with the $\mathbb{E}_{T}$ cut.

Finally, we study the effect of the number of extra dimensions for $M_{P}=3 \mathrm{TeV}$. This dependence is expected to be complicated, as the cross section, multiplicity of the final state particles, and Hawking temperature all depend on $n$ [5]. The six curves for $M_{P}=3 \mathrm{TeV}$ correspond (left to right) to $n=7,5,6,2,3$, and 4 . (As expected, $n=4$ corresponds to the lowest, and $n=7$ to the highest cross section.) The difference between the best and the worst cases corresponds to approximately a factor of two in the integrated luminosity required to achieve a given significance level. The $n=3$ case mostly used in this Letter is on the conservative side.

The $5 \sigma$ discovery of a $130 \mathrm{GeV}$ Higgs boson may be possible with $\mathcal{L} \approx 2 \mathrm{pb}^{-1}, 100 \mathrm{pb}^{-1}, 1 \mathrm{fb}^{-1}, 10 \mathrm{fb}^{-1}$, and $100 \mathrm{fb}^{-1}$ for the fundamental Planck scale of $1,2,3$, 


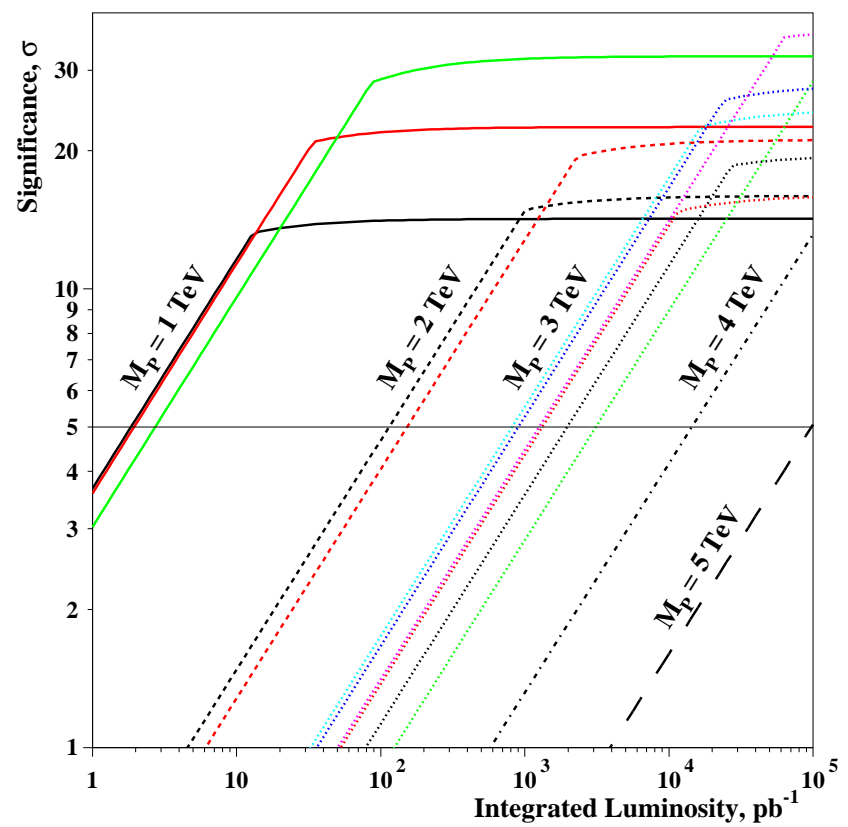

FIG. 3: The significance of a $130 \mathrm{GeV}$ Higgs discovery in the black-hole decays at the LHC as a function of the integrated luminosity. The solid curves correspond to $M_{P}=1 \mathrm{TeV}$, $n=3$ and (left to right) no $b$-tagging, a single $b$-tag, two $b$ tags. The two short-dashed lines correspond to $M_{P}=2 \mathrm{TeV}$, $n=3$, no $b$-tagging, and (left to right) no $\mathbb{E}_{T}$ cut, $\mathbb{E}_{T}<$ $50 \mathrm{GeV}$ cut. The six dotted lines correspond to $M_{P}=3 \mathrm{TeV}$, no $b$-tagging, and (left to right) $n=7,5,6,2,3$, and 4 . The dashed-dotted and long-dashed lines correspond to $M_{P}=4$ and $5 \mathrm{TeV}$, respectively, $n=3$, and no $b$-tagging. The solid horizontal line indicates the $5 \sigma$ discovery level.

4, ahd 5 TeV, respectively. The amount of data required is significantly lower than that in a direct Higgs boson production, if the Planck scale is below $4 \mathrm{TeV}$. While the studies were done for a particular Higgs boson mass, the dependence on the mass is small, and as seen from Figs. 4, 2, a large ramge of masses for which the decay of the Higgs boson into bo domrinates, can be probed. For the Higgs boson masses above $140 \mathrm{GeV}$, the $Z Z^{*}$ decay mode can be employed, similar to the approach used in the direct searches. (The $Z Z^{*}$ mode would result in a significantly reduced combinatorial and in a negligible top quark backgrounds.)

Moreover, the approach outlined in this paper is applicable to searches for other new particles with the masses $\sim 100 \mathrm{GeV}$, particularly the low-scale supersymmetry. Light slepton or top squark searches via this technique may be particularly fruitful, especially since the probability of emitting a spin-0 particle in the $\mathrm{BH}$ decays is expected to be enhanced. Finally, while we considered only the BH production and decay, very similar conclusions apply to the intermediate quantum states, such as string balls [18], which have similar production cross section and decay modes as BHs. In this case, the relevant mass scale is not the Planck scale. but the string scale. whith determines the evaporation tempearature 18 .
Summary: Black hole production at the LHC may change the way we search for new particles. Decays of heavy BHs, tagged with prompt leptons or photons, offer low-background environment for searches of new particles with mass $\sim 100 \mathrm{GeV}$. For example, a $130 \mathrm{GeV}$ SMlike Higgs boson may be observed with the significance of five standard deviations in one hour, day, month, or year of the LHC operation for the values of the fundamental Planck scale of $1,2,3$, and $4 \mathrm{TeV}$, respectively. Thus, future high-energy colliders may become not only the black-hole factories, but factories for new physics.

Acknowledgments: I would like to thank Savas Dimopoulos, Roberto Emparan, and Nemanja Kaloper for valuable discussions. Special thanks to Steve Mrenna for help with the interfacing of the TRUENOIR code with PYTHIA, as well as to the Snowmass 2001 organizers, where these studies have been started. This work was supported partially by the U.S. Department of Energy under Grant No. DE-FG02-91ER40688 and by the Alfred P. Sloan Foundation.

[1] N. Arkani-Hamed, S. Dimopoulos, and G. Dvali, Phys. Lett. B 429, 263 (1998); I. Antoniadis, N. Arkani-Hamed, S. Dimopoulos, and G. Dvali, Phys. Lett. B 436, 257 (1998); N. Arkani-Hamed, S. Dimopoulos, and G. Dvali, Phys. Rev. D 59, 086004 (1999).

[2] P.C. Argyres, S. Dimopoulos, and J. March-Russell, Phys. Lett. B441, 96 (1998) hep-th/9808138.

[3] T. Banks and W. Fischler, JHEP 9906, 014 (1999) hepth/9906038.

[4] R. Emparan, G.T. Horowitz, and R.C. Myers, Phys. Rev. Lett. 85, 499 (2000) hep-th/0003118.

[5] S. Dimopoulos and G. Landsberg, Phys. Rev. Lett. 87, 161602 (2001) hep-ph/0106295.

[6] S.B. Giddings and S. Thomas, e-print: hep$\mathrm{ph} / 0106219 \mathrm{v} 3$.

[7] S.W. Hawking, Commun. Math. Phys. 43, 199 (1975).

[8] M. Carena et al., Report of the Higgs Working Group of the Tevatron Run 2 SUSY/Higgs Workshop, e-print: hep-ph/0010338.

[9] E. Eichten et al., Rev. Mod. Phys. 56, 579 (1984).

[10] A.D. Martin, R.G. Roberts, and W.J. Stirling, preprint RAL-92-078 (1992).

[11] R.C. Myers and M.J. Perry, Ann. Phys. 172, 304 (1986).

[12] S. Dimopoulos and G. Landsberg, in Proc. International Workshop on Future of Particle Physics, Snowmass 2001.

[13] T. Sjöstrand, L. Lönnblad and S. Mrenna, e-print hepph/0108264. We used v6.157.

[14] D.N. Page, Phys. Rev. D 13, 198 (1976).

[15] L. Borissov and J. Lykken, in Proc. International Workshop on Future of Particle Physics, Snowmass 2001.

[16] ATLAS Collaboration, "Calorimeter Performance Technical Design Report," preprint CERN LHCC/96-40.

[17] G. Landsberg and K.T. Matchev, Phys. Rev. D 62, 035004 (2000) hep-ph/0001007.

[18] S. Dimopoulos and R. Emparan, e-print: hep$\mathrm{ph} / 0108060$. 